\title{
Thoracoscopic resection of neurogenic tumours in two children
}

\author{
Mathula Hettiarachchi ${ }^{1}, \mathrm{~KB}$ Galketiya ${ }^{2}$ \\ 'Sirimavo Bandaranaike Specialised Children's Hospital, Department of Surgery, University of Peradeniya, \\ Sri Lanka \\ ${ }^{2}$ Teaching Hospital Peradeniya. Department of Surgery, University of Peradeniya, Peradeniya, Sri Lanka
}

Keywords: VATS; thoracoscopy; children; neuroblastoma; neurogenic tumour

\section{Introduction}

Neurogenic tumours in children account for one-third of all thoracic tumours in children. Out of all the neurogenic tumours, $60 \%$ are malignant, predominantly neuroblastoma[1]. Thoracic neuroblastoma is biologically less aggressive than abdominal neuroblastoma, having excellent long term outcomes.

Conventionally thoracic neurogenic tumours are resected through open thoracotomy but with the availability of miniature endoscopic instruments and improved laparoscopic skills, Video-Assisted Thoracoscopic Surgery(VATS) is increasingly being accomplished with similar long term results[2,3]. Here we report two children who underwent thoracoscopic resection of thoracic neuroblastoma and neurofibroma.

\section{Case presentation 1}

The eight-month-old baby boy was presented with a right supraclavicular soft tissue mass of $3 \mathrm{~cm}$ diameter which was found to be a lymph node mass in computerized tomography (CT) scan. On the CT of the chest, a separate posterior mediastinal mass was noted on the right side. The two masses were not in continuity. An excision biopsy of the neck mass revealed a metastatic deposit of the mediastinal neuroblastoma. The thoracic tumour measured $5 \mathrm{X} 4 \mathrm{X} 4 \mathrm{~cm}$ which was extending up to the hilum of the right lung. $\mathrm{He}$ underwent 3 cycles of neoadjuvant chemotherapy and the tumour was downstaged remarkably. After chemotherapy, the tumour was in a favourable location in the apex of the posterior mediastinum with a size of $3 \mathrm{X} 2 \mathrm{~cm}$.

He underwent thoracoscopic resection of neuroblastoma with a successful outcome. The surgery was done with mainstem left bronchial intubation which enabled complete right lung collapse.

\footnotetext{
Correspondence: Mathula Hettiarachchi

E-mail: drmathula@yahoo.co.uk

(iD) https://orcid.org/0000-0003-0986-5951

Received: 05-11-2020 Accepted: 07-08-2021

DOI: http://doi.org/10.4038/sljs.v39i3.8758

The thoracic cavity was accessed through $5 \mathrm{mmX} 3$ ports, having an optical port at posterior axillary line 5th intercostal space (ICS) with two working ports at the anterior axillary line and posterior to the tip of the scapula. He was positioned in a semi-prone position to expose the lesion of the lung. The mass was located in the apex of the posterior mediastinum wedging the brachiocephalic and subclavian veins. The parietal pleura was circumferentially divided and the lesion was separated from the posterior thoracic wall. The sympathetic chain was divided above and below the lesion and the mass was dissected off the subclavian vessels. The lesion was resected and retrieved through the most posterior port site which was upgraded to $10 \mathrm{~mm}$ size for retrieval..

He recovered uneventfully and was given post-operative chemotherapy after 3 weeks of surgery. He didn't have evidence of recurrence up to the last follow up at 10 months postoperatively.

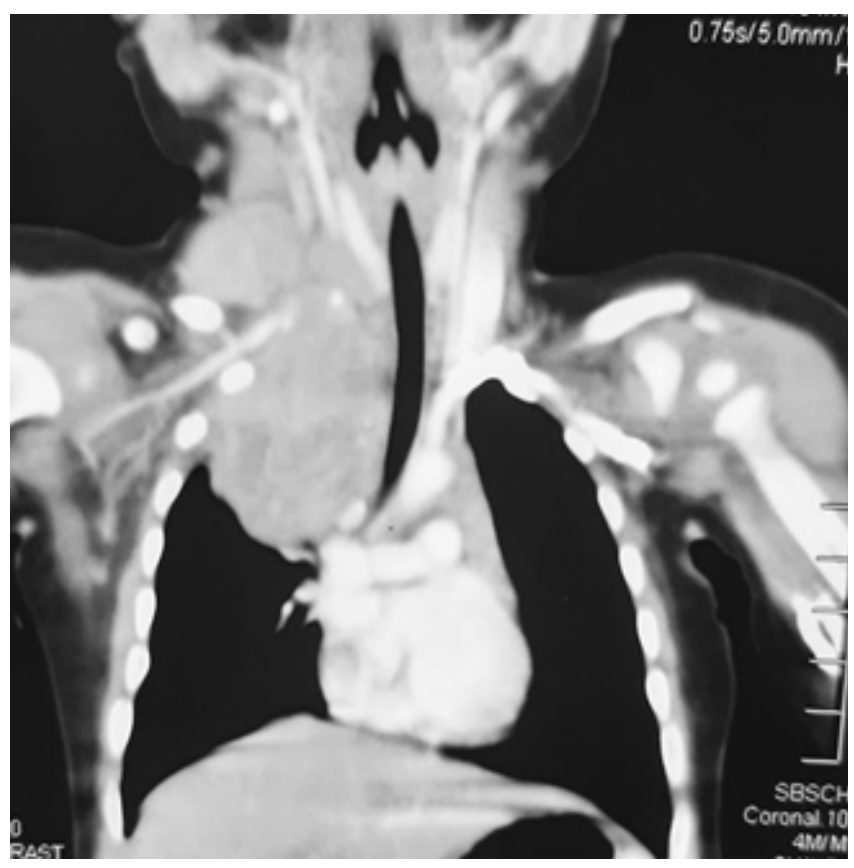

Figure 1. Mediastinal neuroblastoma with the separate neck mass, before chemotherapy. 


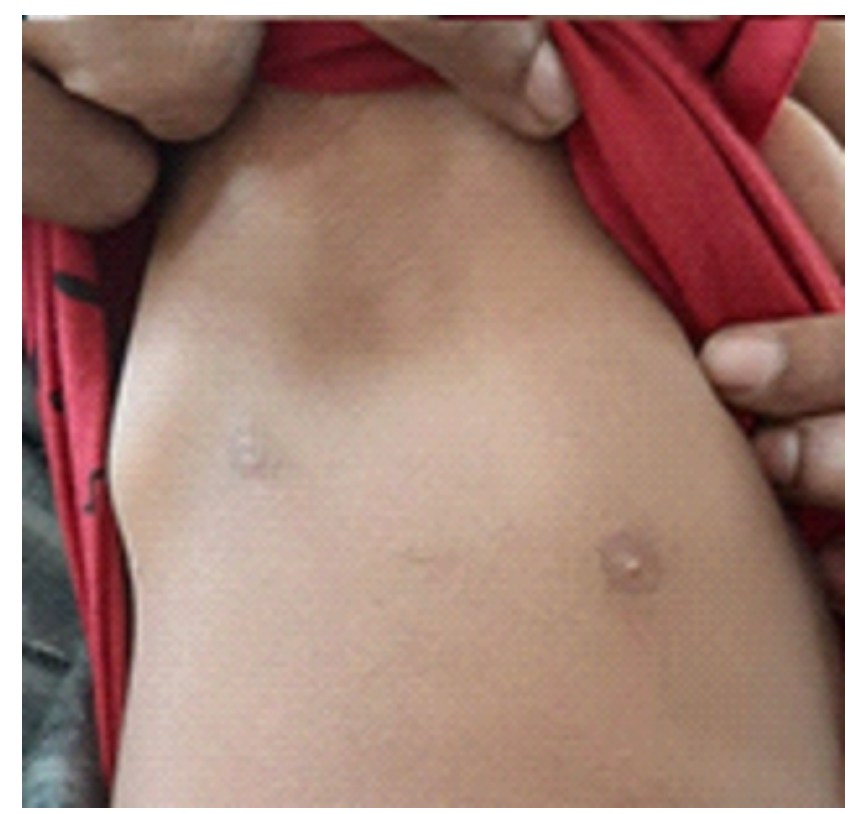

Figure 2. Minimally visible port site scars of the right hemithorax

\section{Case presentation 2}

A 7-year-old boy with hereditary neurofibromatosis type 11 presented with a left cervical mass that was enlarging progressively. There was no pain or tenderness and the lesion was over the left cervical paraspinal space extending from the hyoid bone up to the posterior mediastinum of the left hemithorax. On the CT and Magnetic Resonance Imaging(MRI), it was having a homogenous appearance with tracheal deviation to the right side and compression of the left upper lobe of the lung. The lesion was measuring $12 \mathrm{X} 5 \mathrm{~cm}$ in size.

The cervical lesion was found encircling the left subclavian artery on CT and MRI. The thoracic lesion was $7 \mathrm{X} 5 \mathrm{~cm}$ in size occupying the apex of the posterior mediastinum towards the left side. Trucut biopsy of the cervical part of the lesion revealed features of a benign neurofibroma. Resection was planned due to enlarging nature and the pressure effects of the tumour.

During surgery, cervical mass was accessed lateral to the scalenus group of muscles and mobilised completely. The subclavian artery was skeletonized and looped off the mass at the root of the neck and the upper part of the thoracic mass was mobilised bluntly. Thoracic mass was accessed thoracoscopically with an optical port just below the tip of the scapula and 2 lateral ports at the axilla and 6th ICS at the posterior axillary line. The pleura was divided circumferentially and the mass was elevated off the lateral thoracic wall. Superiorly the mass was dissected off the subclavian vessels. The thoracic mass was completely mobilised and retrieved through the root of the neck. No

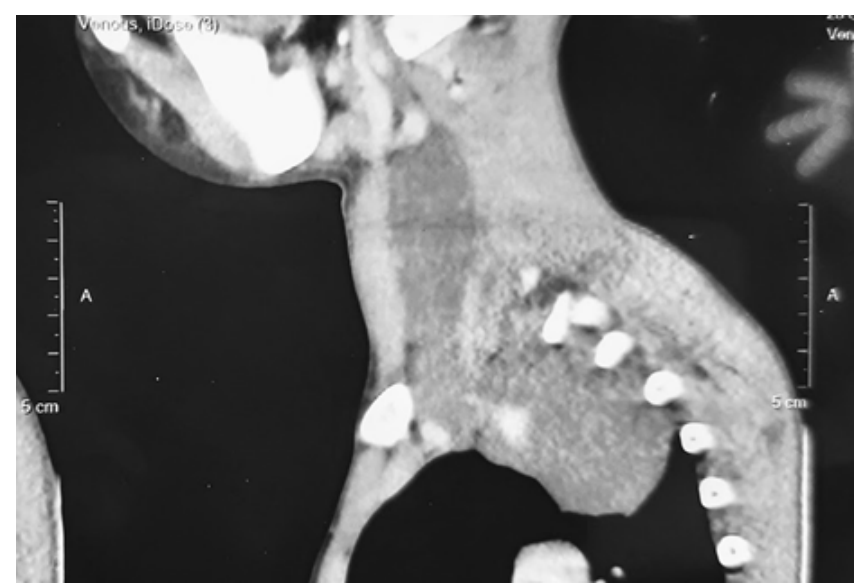

Figure 1. CT scan of the neck and thorax showing the neurofibroma extending from the upper neck up to mediastinum.

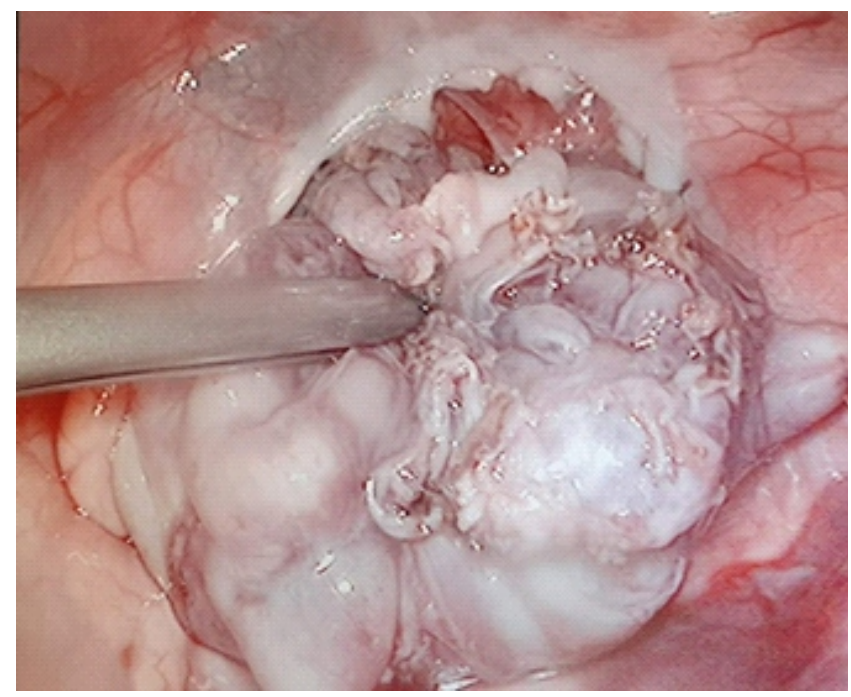

Figure 2. After mobilization of intrathoracic neurofibroma

macroscopic residual lesion was left. The intercostal drain was inserted and removed on day 5.

He was extubated after surgery and was having evidence of partial ptosis which improved on further follow-up. There was transient diaphragmatic paralysis due to phrenic nerve neuropraxia which improved subsequently. Histology confirmed it to be a benign neurofibroma.

\section{Discussion}

Thoracic neurogenic tumours were conventionally resected by thoracotomy but thoracoscopic interventions are increasingly being accomplished to reduce the morbidity of thoracotomy. Morbidity of thoracotomy is due to division of latissimus dorsi and rib spreading during the procedure which could produce long term disability in shoulder movements and spinal deformity.[4] In children, $60 \%$ of thoracic neurogenic tumours are malignant, hence thoracoscopic 
interventions are still controversial due to the feared risk of inadequate resections with positive surgical margins and port site recurrence which has been rarely reported at least in adults.[5] With the availability of miniature instruments and increasing experience in paediatric thoracoscopy, improved outcome and effectiveness has been shown in thoracoscopic resection of these tumours in the literature. [6] In a recent review, it has been stated that the laparoscopic or thoracoscopic approach to neuroblastoma has an equal outcome compared to the open approach, given the adequacy of oncological resection[7].

Contralateral main stem bronchial intubation has been conventionally used in thoracoscopic procedures but for smaller tumours, tracheal intubation and lung collapse achieved with $\mathrm{CO} 2$ insufflation have been successfully used. In the first patient, the lung collapse was achieved with main stem bronchial intubation while the second surgery was done with tracheal intubation and $\mathrm{CO} 2$ insufflation with low flow (11/min) and low pressure (4mmgh) in the pleural cavity. In most thoracoscopic interventions, 3 ports had been used in triangulation with a camera inserted at the convenient port site to improve visualization. Magnification of view in thoracoscopy greatly assists in dissection and achieving hemostasis compared to open surgery, especially when the tumour is in the apical region.

All authors disclose no conflict of interest. The study was conducted in accordance with the ethical standards of the relevant institutional or national ethics committee and the Helsinki Declaration of 1975, as revised in 2000 .

\section{References}

1. King DR, Groner JI, Teich S. Mediastinal cysts and tumors. In: Ziegler MZ, Azizkhan RG, Weber TR, editors. Operative pediatric surgery. New York (NY): McGraw-Hill; 2003. p. 40719.

2. Malek MM, Mollen KP, Kane TD, Shah SR, Irwin C. Thoracic neuroblastoma: a retrospective review of our institutional experience with comparison of the thoracoscopic and open approaches to resection. Journal of pediatric surgery. 2010 Aug 1;45(8):1622-6.. https://doi.org/10.1016/j.jpedsurg.2010.03.018

3. Lacreuse I, Valla JS, de Lagausie P, Varlet F, Héloury Y, Temporal G, Bastier R, Becmeur F. Thoracoscopic resection of neurogenic tumors in children. Journal of pediatric surgery. 2007 Oct 1;42(10):1725-8.

https://doi.org/10.1016/j.jpedsurg.2007.05.030

4. Kucukarslan N, Kirilmaz A, Arslan Y, Sanioglu Y, Ozal E, Tatar H. Muscle sparing thoracotomy in pediatric age: a comparative study with standard posterolateral thoracotomy. Pediatric surgery international. 2006 Oct 1;22(10):779-83.

https://doi.org/10.1007/s00383-006-1776-7

5. Pentek F, Schulte JH, Schweiger B, Metzelder M, Schündeln MM. Development of Port-Site Metastases Following Thoracoscopic Resection of a Neuroblastoma. Pediatric blood \& cancer. 2016 Jan;63(1):149-51. https://doi.org/10.1002/pbc.25677

6. Rothenberg SS. Thoracoscopy in infants and children: the state of the art. Journal of pediatric surgery. $2005 \mathrm{Feb}$ 1;40(2):303-6. https://doi.org/10.1016/j.jpedsurg.2004.10.021

7. Van Arendonk KJ, Chung DH. Neuroblastoma: tumor biology and its implications for staging and treatment. Children. 2019 Jan;6(1):12.). https://doi.org/10.3390/children6010012

\section{Learning Points:}

- Resection of intrathoracic neurogenic tumours in children is safe and effective with thoracoscopic techniques even in malignant tumours.

- In addition to all advantages of minimal access surgery, thoracoscopic resections have shown less morbidity by avoiding rib spreading thoracotomy and precision in surgical technique due to greater access and magnification. 